\title{
EXPERIENCIA DE VIDA PROFESIONAL DEL DR. ROBERTO LLANOS ZULOAGA
}

\author{
Experience of Dr. Roberto Llanos Zuloaga life
}

Jenny Quezada Zevallos*

\begin{abstract}
Resumen
La biografía del Dr. Roberto Llanos Zuloaga, se realizó contando con su autorización, habiéndose realizado dos entrevistas en agosto y septiembre del año 2010, en ellas pudimos disfrutar de su sentido de humor, cordialidad, energía de vida y sabiduría. Después de terminadas cada una de las entrevistas, surgió en mi una necesidad de escribir en forma poética, mis sentimientos y sensaciones, conformando una respuesta estética a la experiencia vivida. Se incluyen recensiones de sus libros.
\end{abstract}

\section{INTRODUCCIÓN}

A puertas de que la Facultad de Psicología y Humanidades de la Unifé, cumpla 50 años de funcionamiento, consideramos necesario hacer conocer la vida de las personas y personajes de la facultad, que con sus talentos personales y profesionales, han aportado al desarrollo de la misma y han permitido que a la fecha el prestigio de nuestra facultad nos ubique en los primeros lugares como centro formador de psicólogas.

El Dr. Roberto Llanos Z. catedrático con más de 35 años de permanencia en nuestra casa de estudio, años durante los cuales asumió los cargos de Jefe de Departamento y Coordinador del Doctorado de Psicología, ha dejado en cada una de las que fuimos sus estudiantes una estela de enseñanzas didácticas, éticas y de organización personal y profesional, trasmitiendo su vitalidad y fortaleza manifestada en el día a día y en sus obras.

\section{VIDA PERSONAL Y PROFESIONAL}

Nació en Lima - Perú el 8 de julio de 1933, es el cuarto hijo de ocho. Realizó sus estudios en el Colegio Emblemático Nacional de Guadalupe. Sus intereses

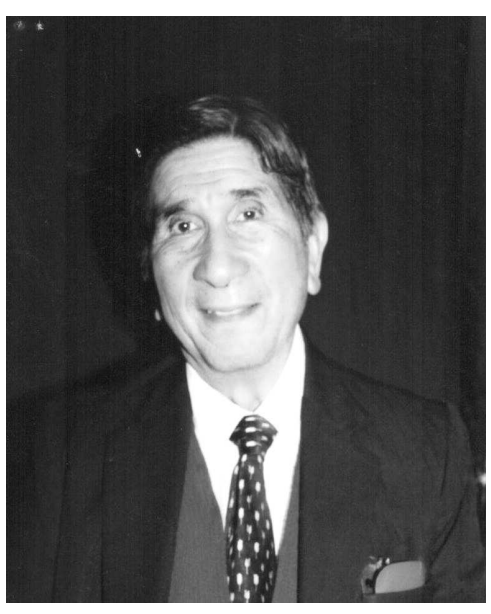

abarcan la música (en especial la ópera), interés compartido con su padre, y la literatura, con la cual obtuvo dos premios internacionales por los poemas "Campana de la Libertad de Huaura" y "Vida y obra del Generalísimo Francisco de Miranda - Libertador de Venezuela".

Su inclinación por la Psicología surge a raíz de asistir a unas charlas sabatinas que daba el Dr. Alberto Seguín, sobre algunos aspectos de la psicoterapia, en el auditorio del Hospital Obrero - hoy conocido como el Hospital Guillermo Almenara. Su perseverancia lo llevó a escucharlas durante seis meses. Al culminar sus estudios secundarios, encuentra que no existían los estudios de Psicología en el Perú, y decide estudiar Medicina para poder dedicarse a la psiquiatría: "ahí voy a escribir muchos libros" (entrevista agosto 2010).

Ingresa a la Universidad Nacional Mayor de San Marcos, y practica en el Hospital Santo Toribio de Mogrovejo, como ayudante del Dr. Saavedra, quien fuera asistente del Dr. Honorio Delgado. En dicha

\footnotetext{
* Docente de la Facultad de Psicología y Humanidades de la Universidad Femenina del Sagrado Corazón. jenquezada@unife.edu.pe
} 
institución, realizarón experimentos psiquiátricos con la sustancia Liserg Saure Dietylamid LSD o Dietilamida del ácido lisérgico. Posteriormente, realiza prácticas con el Dr. Saavedra en el pabellón 20 del Hospital Larco Herrera y, en el último año de la carrera, las cumple en el Hospital de la Policía. En esa época, se reunía con un grupo de psiquiatras y médicos y conformaron la Sociedad Amantes de la Neuropsiquiatría que funcionó durante 20 años.

Su primer trabajo como psiquiatra lo lleva a cabo en el Hospital de la Policía y, como docente en la Facultad de Medicina de Trujillo (1963), donde laborará con el Dr. Grover Mori, quien fuera también alumno de Honorio Delgado. En 1966, viaja a Alemania a seguir estudios de post grado en Tubingen, en la especialidad de psicoterapia, con especial mención en psicodrama; fue presentado a dicha institución por el Dr. Honorio Delgado. Luego, de 1967 a 1968, fue asistente en el Instituto Max Planck para Psiquiatría de Munich.

En Alemania, contrae matrimonio con una pedagoga alemana. A fines de 1968, regresa al Perú con su esposa y dos hijos. Se incorpora al Ministerio de Salud, al servicio de Psiquiatría Infantil del Hospital San Bartolomé de Lima, el cual estuvo dirigiendo durante 12 años. También, empieza a trabajar en la Universidad Femenina del Sagrado Corazón, en la Facultad de Psicología, enseñando los cursos de Caracterología, Psicología de la Personalidad entre otros. Paralelamente, es docente en las facultades de Psicología de la Universidad Ricardo Palma, Pontificia Universidad Católica del Perú, Universidad de Lima, Universidad San Martín de Porres, Universidad Nacional Mayor de San Marcos y de la Escuela de Enfermería del Hospital Loayza, completando su labor en el Instítuto Nacional de Salud Mental Honorio Delgado Hideyo Noguchi en Lima, del cual fue médico fundador y organizador.

En 1975, pasa a ser miembro del staff del consultorio de Psiquiatría en la Clínica Ricardo Palma. Se gradúa como Doctor en Medicina en 1990 en la Universidad Peruana Cayetano Heredia. Es miembro de la Asociación Peruana de Bioética, Asociación Peruana de Psicoterapia, Asociación Peruana de Psiquiatría y Sociedad Peruana de Psiquiatría Biológica.
Ha ejercido cargos importantes en instituciones médicas y educativas del país. En 1961, fue Presidente de la Sociedad Peruana de Psiquiatría, Neurología y Neurocirugía, Vicepresidente de la Asociación Psiquiatrica Peruana (1984-1985) y Presidente de la misma en los períodos 1986,1987 y 1988, Jefe de Departamento de la Facultad de Psicología de la Universidad Femenina del Sagrado Corazón, Coordinador del Doctorado de la misma Universidad de 1996 al 2003

\section{PUBLICACIONES REALIZADAS}

\section{El matrimonio simbiótico (1992)}

En este libro, el autor presenta los instrumentos fundamentales del Análisis Transaccional haciendo hincapié en el Análisis Estructural y Funcional de los estados del "Yo", las Transacciones, las Caricias, la Posición Existencial y el Argumento de Vida. Asimismo, ofrece los resultados del estudio de 20 parejas con problemas de relación interpersonal, estudiados en la consulta psiquiátrica ambulatoria de una clínica privada de Lima. La muestra está conformada por matrimonios peruanos de clase media alta, cuyas edades fluctúan entre 24 y 55 años. La muestra se dividió en cuatro grupos significativos:

1. Matrimonios que viven juntos bajo una relación comportamental simbiótica.

2. Matrimonios separados legalmente o divorciados, con incapacidad de separarse físicamente manteniendo la misma forma comportamental simbiótica previa.

3. Matrimonios que viven con terceras personas adultas, parientes o no con relación homosexual o sin ella, en la que se alternan las relaciones comportamentales simbióticas.

4. Matrimonios, en los que uno o ambos cónyuges mantienen relaciones amorosas heterosexuales con terceras personas alternando la relación comportamental.

En la muestra estudiada, se hallaron los siguientes síntomas: descalificación, grandiosidad, escalamiento, conducta pasivo no productiva (CPCNP), sobreadaptación, agitación, incapacidad y violencia, "Padre Salvador - "Niño adaptado sumiso", "Padre Crítico - "Niño Adaptado Rebelde" y cónyuges con 
"Padres Críticos". El 50\% del total de la muestra muestran síntoma simbiótico.

\section{El encuentro grupal (1993)}

El libro está conformado por 13 capítulos, y un apéndice. En el primer capítulo, el autor desarrolla las principales características de los grupos de encuentro, la clasificación de las terapias grupales, las diferencias entre terapia grupal y terapia familiar, las estructuras de los grupos y la técnica del encuentro grupal. En los siguientes, las bases teóricas, metodología y diferentes técnicas: Análisis Transaccional, Gestáltica, Psicodrama, Grito primario, Bionergética, Grupo Balint, Rolfing, Grupo Alfa, Relajación Progresiva, Terapias Grupales de Relajación y Grupo de Interacción en un tema. En el apéndice, se halla una compilación de técnicas seleccionadas por A. Castillo.

\section{Personalidad y carácter. Estructura y evaluación (1994)}

El libro está organizado en dos capítulos I La estructura de la Personalidad y del carácter y II Evaluación de la personalidad. A lo largo del primer capítulo desarrolla la definición, evolución, estructura del temperamento, clasificación, finalizando con el estudio de la estructura personal según Krestschmer, Sheldon, Guilford, Freíd, Adler, Horney y Lersch.

En el segundo capítulo desarrolla de manera detallada la evaluación de la personalidad según la teoría Psicofisiológica, la teoría "Nueva Era", así como según la clasificación DSM-IV e ICD-10, continuando con la evaluación de la personalidad psicopática, borderline, esquizoide, delictiva, violenta y depresiva.

\section{Psicopatología del niño y del adolescente Veinte Lecciones (1996)}

En esta obra, el autor desarrolla los principales temas de la psiquiatría infantil: epidemiología y prevención psicopatológica infantil y psicopatología infantil en el Perú. Analiza los principales trastornos mentales de inicio en la niñez y adolescencia: angustia infantil, depresión infantil y retardo mental, autismo y esquizofrenia infantil, enuresis, encopresis, hiperkenesis, sexualidad infantil, anorexia nerviosa infanto juvenil, la enfermedad de Pilles de La Tourette y un resumen de las principales clasificaciones de las enfermedades mentales DSM-IV y ICD-10. Considera los aportes del Análisis Transaccional en la mejor comprensión de la psicopatología infantil, revisando los temas de matrimonio simbiótico y psicopatología infantil, reparentalización y psicopatología infantil, redecisión y divorcio.

Ofrece una revisión de técnicas necesarias en el trabajo en psicopatología; nos referimos a la anamnesis, la primera entrevista con los padres y la primera entrevista psicoterapéutica infantil. Completa esta obra con un marco teórico de los niveles de desarrollo según Erick Erickson y otras escuelas

\section{Musicoterapia Diez Lecciones (1999)}

El autor desarrolla en la primera parte del libro los fundamentos de la musicoterapia: recuerdo, relajación, respiración, argumento, biografía, instrumento, base histórica, base médica, base psicológica y base social. En la segunda parte, plantea dos talleres de musicoterapia; el primero de Estimulación y Motivación y el segundo de Relajación y meditación. Plantea las aplicaciones de la Musicoterapia en psicoterapia, rehabilitación, educación y aprendizaje. El libro culmina con un artículo sobre Musicoterapia para niños escrito por Sofía Llanos.

Ha escrito más de 70 artículos publicados en revistas nacionales e internacionales sobre diferentes temas.

He considerado necesario incluir los poemas que surgieron después de cada una de las entrevistas realizadas con el Dr. Roberto Llanos

\section{PRIMER ENCUENTRO CON EL MAESTRO}

He iniciado el viaje

de conocerte maestro;

hoy fuimos caminando

por tus recorridos

entre la granja y el Mercado Central

Estuvimos en la compra con lo ganado

de una novela, dos, tres;

cada semana una más, 
hasta que sumaron más de 100.

Tenías 12 años y amabas escribir y leer.

De un padre amante de la opera y la literatura, ¿qué se podía esperar?

Te inoculó en largas conversaciones

el bicho de la música y el placer de viajar.

Ser cuarto hermano de 7 hijas mujeres,

fácil, fácil, no debió ser;

quién lo supiera, maestro, que entrenándote estabas para trabajar en la Unifé.

Escuchando a Seguín, conociste la psíque y los juegos intrincados que en la mente se dan.

Caminado te ibas preguntando

¿Cómo unir literatura y psíque?

Y un día te contestaste

¡Eureka!

!psiquiatra he de ser!

El azar, unos dirán;

otros, "la suerte loca",

te hizo ir encontrando en tu camino

al Dr. Saavedra, al Dr. Moorí y al Dr. Honorio Delgado; entre muchos más.

Mente joven, cuerpo ágil, que arremete, sin saber toda, toda su energía en aprender.

Alemania te esperaba

con un idioma ¡Dios mío, qué difícil de aprender!

Entonces, te dijiste:

"Si lo haces cada día, cada noche,

cada mes, irás comprendiéndolo,

dominándolo al derecho y al revés".

La música te jalaba,

la ópera pudo más.

Te veo tres horas antes,

en tu cola, esperar que, en el Teatro de Munich,

te vendan el ticket por casi un real.

Así como el pez regresa a las aguas

de las cuales salió,
Lima te hacia señas, gestos y coqueteos

que duraron cinco años

antes de regresar.

Y por cosas de la vida,

la Psicología te fue a encontrar,

con un abrazo fraterno,

como docente de una universidad.

¡Perdón, me he equivocado!

¡No fue una, fueron siete!

¡Nada más!

Finalmente, en la lidia,

quedó solo una,

una y nada más.

Buscando respuestas a lo observado,

el AT te fue ganando,

un pasito cada vez,

cual vals que, secretamente, se baila soñando,

despierto o puesto al revés.

La Bioética fue a tu encuentro,

como la abeja a la miel.

Te acompaño, ¡oh, maestro!,

en esta tu historia de maestro,

de psiquiatra, escritor

y qué sé yo de qué más.

Nos queda aún camino para conversar

de los otros caminantes,

que ajustaron su andar

a tu prisa, movimientos, a eso y a tu enfermedad

Nos vemos, maestro

Nos vemos, el día lunes será.

Lima, agosto 2010

\section{SEGUNDO ENCUENTRO CON EL MAESTRO}

Hoy hablamos de la esposa, hijos,

de aquellos de quienes orgulloso estás.

No es para menos maestro,

Con un hijo pediatra

y una hija psicóloga y educadora, además. 
Me hablaste de los nietos, sonriendo sin parar, en Alemania, además de ciencia, esposa hallaste a la par.

Es bueno estar acompañado en esta, tu aventura de dar y de andar.

Recuerdas, ya con pena, ya con risa, que el $10 \%$,

no más, cumplía con los trabajos a cabalidad.

¿Qué decir del 90\% restante?

En ellas, la semilla está, y, a su ritmo y a su tiempo, algún fruto habrán de dar

Paciencia, maestro, paciencia.

El futuro nos evidenciará lo sembrado, en tus alumnas de aquí y de allá.

Prestabas tu biblioteca:

de eso, nunca me he de olvidar;

pues yo, golosa de libros,

de todos y cada uno,

algo recogí por azar y necesidad.

¿Qué experiencia la tuya, maestro!,

de regalar libros por demás!

Y a la vuelta de una luna, estos regresar,

porque sitio no hay para ellos,

allí, en el lugar que elegiste para donar.

La exactitud en el tiempo acordado,

la planificación escrupulosa del momento de compartir.

Tus valores se inhalan,

como lo haces con una flor al pasar:

ellos están presentes,

allí donde tú estás.

Respeto, caballerosidad,

orden y cumplimiento,

eso es lo que he sentido al contacto contigo estar.

Muchas veces, querido maestro,

me he preguntado

Fecha de recepción: 30 de setiembre, 2011

Fecha de aceptación: 20 de octubre, 2012
¿Cuál es el origen de tu tenacidad,

tu resistencia,

tu lucha frente a la enfermedad?

He escuchado a otros,

de tus clases en la cama dar;

ahora me toca a mí, maestro,

eso también presenciar.

Cuéntame, en secreto, maestro,

el origen de tu vitalidad,

de tu amor por el trabajo,

de tu pasión por dar.

Levantando la mano derecha,

tu secreto prometo guardar,

en medio de jeroglíficos,

cuentos, chistes y demás.

Sí alguien logra descifrar,

pese al uso de mis artilugios al tu vida relatar, que quede clarito, maestro,

que el que está listo para mirar, mirará.

Septiembre, 2010

\section{REFERENCIAS}

Llanos Zuloaga Roberto (1992) El matrimonio simbiótico. Lima: Libro Amigo

Llanos Zuloaga Roberto (1993) El encuentro grupal. Lima: Libro Amigo

Llanos Zuloaga Roberto (1994) Personalidad y carácter. Estructura y evaluación. Lima: Libro Amigo

Llanos Zuloaga Roberto (1996) Psicopatología del niño y del adolescente Veinte Lecciones. Lima: Libro Amigo

Llanos Zuloaga Roberto Musicoterapia Diez Lecciones (1999) Lima: ADIG

Quezada Zevallos Jenny (2010) Entrevista al Dr. Llanos Z. Lima, 24 de agosto y 7 de setiembre 2010.

Agradecimiento especial a la Mg. Rosa Dodobara por su apoyo en la revisión del artículo. 